\title{
Repetitive hyperbaric oxygen treatment increases insulin sensitivity in diabetes patients with acute intracerebral hemorrhage
}

This article was published in the following Dove Press journal:

Neuropsychiatric Disease and Treatment

10 February 2017

Number of times this article has been viewed

\section{Qian Xu \\ Yi-ting Wei \\ Shuang-bo Fan \\ Liang Wang \\ Xiao-ping Zhou}

Department of Brain Surgery, Ningbo

Zhenhai Longsai Hospital, Zhejiang,

People's Republic of China
Correspondence: Qian Xu

Department of Brain Surgery, Ningbo Zhenhai Longsai Hospital, No 356 Shengli Rd, Zhenhai District, Ningbo, Zhejiang, 315200, People's Republic of China Email xuqian652I@I26.com
Aim: The role of hyperbaric oxygen therapy (HBOT) in the treatment of acute ischemic stroke is controversial. This study aims to investigate whether the peripheral insulin sensitivity of type 2 diabetes patients suffering from intracerebral hemorrhage can be increased after HBOT.

Methods: Fifty-two type 2 diabetes participants were recruited after being diagnosed with intracerebral hemorrhage in our hospital. Insulin sensitivity was measured by the glucose infusion rate during a hyperinsulinemic euglycemic clamp $\left(80 \mathrm{mU} \mathrm{m}^{-2} \mathrm{~min}^{-1}\right)$ at baseline and 10 and 30 days after HBOT sessions. Serum insulin, fasting glucose, and hemoglobin $\mathrm{Al}_{\mathrm{C}}$ were measured in fasting serum at baseline and after HBOT sessions. In addition, early ( $\sim 10$ days after onset) and late (1 month after onset) outcomes (National Institutes of Health Stroke Scale, NIHSS scores) and efficacy (changes of NIHSS scores) of HBOT were evaluated.

Results: In response to HBOT, the glucose infusion rate was increased by $37.8 \% \pm 5.76 \%$ at 1 month after onset compared with baseline. Reduced serum insulin, fasting glucose, and hemoglobin $\mathrm{A}_{\mathrm{C}}$ were observed after HBOT. Both early and late outcomes of the HBOT group were improved compared with baseline $(P<0.001)$. In the control group, there was significant difference only in the late outcome $(P<0.05)$. In the assessment of efficacy, there were statistically significant differences between the groups when comparing changes in NIHSS scores at 10 days and 1 month after onset $(P<0.05)$.

Conclusion: Peripheral insulin sensitivity was increased following HBOT in type 2 diabetes patients with intracerebral hemorrhage. The HBOT used in this study may be effective for diabetes patients with acute stroke and is a safe and harmless adjunctive treatment.

Keywords: hyperbaric oxygen therapy, type 2 diabetes, intracerebral hemorrhage, NIHSS, glucose infusion rate

\section{Introduction}

Hyperbaric oxygen therapy (HBOT) represents a possible therapy for both acute ischemic stroke and intracerebral hemorrhage. The potential benefits of HBOT include increased oxygen delivery, decreased cerebral edema, decreased lipid peroxidation, inhibition of leukocyte activation, and maintenance of blood-brain barrier integrity. ${ }^{1-3}$ HBOT was proved to reduce the volume of brain infarction and improve outcome in both mice and rat stroke models with hyperglycemia or diabetes. ${ }^{4-6}$ Despite beneficial results in several animal models, ${ }^{7,8}$ the effectiveness in human stroke is still controversial. It was reported that a 56-year-old Chinese patient suffering from acute ischemic stroke on the left corona radiata leading to right hemiparesis and dysarthria had great improvement after repetitive (10 dives) HBOT with lower pressure (2.0 ATA) and 
short duration $(60 \mathrm{~min}) .{ }^{9}$ In recent years, there have been a few randomized controlled trials in humans as well, but with different strategy as control. ${ }^{10-12}$

This study aims to determine the insulin-sensitizing effect of HBOT in type 2 diabetes patients suffering from intracerebral hemorrhage. In addition, the efficacy, safety, and feasibility of hyperbaric oxygen at 2.5 ATA were prospectively evaluated.

\section{Methods}

This study was a randomized, prospective, normobaric oxygen therapy (NBOT)-controlled pilot study to investigate the potential effect of HBOT on peripheral insulin sensitivity in type 2 diabetes patients with intracerebral hemorrhage. Subjects were randomized according to their time stratum to receive either HBOT or NBOT. Treatment designation was placed in a sealed envelope and seen only by the hyperbaric nurse at the time of treatment. This study was approved by the Zhejiang Medical Institutional Ethics Committee. Before enrollment, written informed consent was obtained from the patients or their next of kin prior to initiation of any clinical procedures that were performed solely for the purpose of determining eligibility for research.

\section{Participants}

Participants included adults aged 18 years presenting to an emergency department within $24 \mathrm{~h}$ after stroke onset with a measurable deficit on the National Institutes of Health Stroke Scale (NIHSS) and with evidence of hemorrhage on computerized tomography (CT) scan. Patients were excluded if they were not diabetes patients before onset, had a stroke within 3 months, had an NIHSS score $>22$, or died within the whole period of 6 months of follow-up. Patients were also excluded if they had risk factors for HBOT, including a history of sickle cell disease, bowel obstruction, severe chronic obstructive pulmonary disease, pneumothorax, or evidence of cardiac arrhythmia deemed by an investigator as potentially mandating emergent intervention.

\section{Interventions}

Fifty-two type 2 diabetes subjects were randomized to receive either HBOT $(n=23)$ or NBOT $(n=29)$. The HBOT group underwent a 60-min continuous treatment once a day for 30 days in a monoplace HBOT chamber pressured with $100 \%$ oxygen to 2.5 ATA (50 ft of seawater). The NBOT group underwent similar treatment except with a pressure of
1.5 ATA (4.48 ft of seawater). The NBOT group treatment was designed to simulate pressure changes within the tympanic membrane.

\section{Outcome measures}

Testing was undertaken at approximately the same time each morning, and sampling was undertaken at a similar time each visit. Baseline assessments were performed before intervention. Early ( $\sim 10$ days after onset) and late (1 month after onset) outcomes (neurobehavioral NIHSS scores) and efficacy (changes in NIHSS scores) of HBOT were evaluated. The criteria of NIHSS score are displayed in detail in Figure 1.

The 3.5-h hyperinsulinemic euglycemic clamp was performed at baseline, 1 month and 6 months after onset. All tests were performed in normobaric room air, outside the hyperbaric chamber, as previously described. ${ }^{13}$ Briefly, two intravenous cannulae were inserted into veins on opposite arms. One cannula was connected to an infusion of insulin at a fixed rate of $80 \mathrm{mU} \mathrm{m}^{-2} \mathrm{~min}^{-1}$, together with a variable-rate infusion of $25 \%$ dextrose. The other cannula allowed blood sampling every $5-10$-min to assess blood glucose levels by a hand-held glucometer. The target blood glucose level was $6 \mathrm{mmol} \mathrm{L}^{-1}$. Insulin sensitivity was calculated from the glucose infusion rate (GIR) at the end of the 3.5-h clamp. In addition, serum insulin and hemoglobin A1c (HbA1c) were measured. To avoid any physical effort that might influence glucose uptake, the participants remained sedentary in a chair.

\section{Statistical analysis}

SPSS version 16.5 was used to perform all statistical analyses. Baseline patient characteristics were compared using either the Wilcoxon rank-sum test or Fisher's exact test. Baseline categorical variables were analyzed by Fisher's exact test, whereas numerical variables were analyzed by Student's $t$-test (equal variance test). Differences between groups were analyzed using one-way analysis of variance (ANOVA). To compare paired data, the Shapiro-Wilk test and the Wilcoxon signed-rank test were used to test the data of clinical responses. Variables in both groups (eg, changes in NIHSS scores) were compared by equal variance test for early efficacy and by the Mann-Whitney $U$-test for late efficacy. A significance level of $P<0.05$ was used for determining statistical significance.

\section{Results}

From August 2012 to June 2016, 52 patients were recruited, including 23 in the HBOT group and 29 in the control group. 


\begin{tabular}{|c|c|c|}
\hline IA Level of consciousness & IB Orientation questions (two) & IC Response to commands (two) \\
\hline 0 alert & 0 both correctly & 0 both correctly \\
\hline I drowsy & I one correctly & I one correctly \\
\hline 2 obtunded & 2 neither correctly & 2 neither correctly \\
\hline \multicolumn{3}{|l|}{3 coma/unresponsive } \\
\hline 2 Gaze & 3 Visual fields & 4 Facial movements \\
\hline 0 normal horizontal movements & 0 no visual field defect & 0 normal \\
\hline I partial gaze palsy & I partial hemianopia & I minor facial weakness \\
\hline \multirow[t]{2}{*}{2 complete gaze palsy } & 2 complete hemianopia & 2 partial facial weakness \\
\hline & 3 bilateral hemianopia & 3 complete unilateral palsy \\
\hline 5 Motor function (arm) & 6 Motor function (leg) & 7 Limb ataxia \\
\hline 0 no drift & 0 no drift & 0 no ataxia \\
\hline I drift before 10 seconds & I drift before 5 seconds & I ataxia in one limb \\
\hline 2 falls before 10 seconds & 2 falls before 5 seconds & 2 ataxia in two limbs \\
\hline 3 no effort against gravity & 3 no effort against gravity & \\
\hline 4 no movement & 4 no movement & \\
\hline 8 Sensory & 9 Language & 10 Articulation \\
\hline 0 no sensory loss & 0 normal & 0 normal \\
\hline I mild sensory loss & I mild aphasia & I mild dysarthria \\
\hline \multirow[t]{2}{*}{2 severe sensory loss } & 2 severe aphasia & 2 severe dysarthria \\
\hline & 3 mute or global aphasia & \\
\hline \multicolumn{3}{|l|}{ I I Extinction or inattention } \\
\hline \multicolumn{3}{|l|}{0 absent } \\
\hline \multicolumn{3}{|c|}{ I mild (loss of one sensory modality) } \\
\hline 2 severe (loss of two sensory mo & & \\
\hline
\end{tabular}

Figure I The criteria of NIHSS score in detail.

Abbreviation: NIHSS, National Institutes of Health Stroke Scale.

Their baseline properties and clinical characteristics were compared and analyzed, with no statistically significant difference between the two groups in each item (gender, age, body mass index, triglycerides, total cholesterol, and homocysteine, as shown in Table 1).

A significant increase was observed in the change in insulin sensitivity during the HBOT sessions (Figure 2A, 0 day vs 30 days: $37.2 \pm 5.8$ vs $51.2 \pm 4.9, P<0.05$ ). Conspicuous decreases in the changes in fasting glucose, insulin, and HbAlc were also observed in the HBOT group after 30-day sessions (fasting glucose: $11.3 \pm 1.5$ vs $9.6 \pm 1.1, P<0.05$; insulin: $22.9 \pm 4.1$ vs $17.8 \pm 2.6, P<0.05$; HbA1c: $9.2 \pm 1.6$ vs $7.8 \pm 1.3, P<0.05$; Figure 2B-D).

In the HBOT group, the paired $t$-test was used to compare the NIHSS scores before and after HBOT in each group. The NIHSS scores were prominently decreased at both 10 days (mean $\pm \mathrm{SD}, 9.2 \pm 1.6)$ and 1 month after HBOT $(8.1 \pm 0.8)$ compared to the pre-HBOT data at baseline (13.5 \pm 2.2 , $P<0.01$; Figure 3B). In the control group, the scores were evaluated as an obvious decrease only at 1 month after HBOT compared with baseline $(8.7 \pm 2.1$ vs $12.1 \pm 2.7, P<0.05$; Figure $3 \mathrm{~A})$. The difference in baseline NIHSS scores between

Table I Baseline characteristics in two groups

\begin{tabular}{|c|c|c|c|}
\hline Indicators & NBOT $(n=29)$ & HBOT $(n=23)$ & $P$-value \\
\hline Age, median (range) & $69(44-85)$ & $65(40-83)$ & 0.95 \\
\hline Female, n (\%) & $9(31.0)$ & $8(34.8)$ & 0.99 \\
\hline Body mass index $\left(\mathrm{kg} / \mathrm{m}^{2}\right)$ & $30.6(27.7-34.9)$ & $30.9(30.1-35.7)$ & 0.99 \\
\hline NIHSS score, median (range) & $8(3-17)$ & $7(2-20)$ & 0.93 \\
\hline Glucose (mmol/L) & $9.8(8.1-13.0)$ & $9.3(7.7-12.8)$ & 0.95 \\
\hline Triglycerol (mg/dL) & 137 (54-388) & $165(57-419)$ & 0.86 \\
\hline Total cholesterol (mg/dL) & 183 (1 I 8-292) & $202(\mid 24-28 I)$ & 0.82 \\
\hline Homocysteine ( $\mu \mathrm{mol} / \mathrm{L})$ & I I.8 (5.9-|6.2) & I I.3 (3.3-20.6) & 0.98 \\
\hline
\end{tabular}

Abbreviations: NBOT, normobaric oxygen therapy; HBOT, hyperbaric oxygen therapy; NIHSS, National Institutes of Health Stroke Scale. 

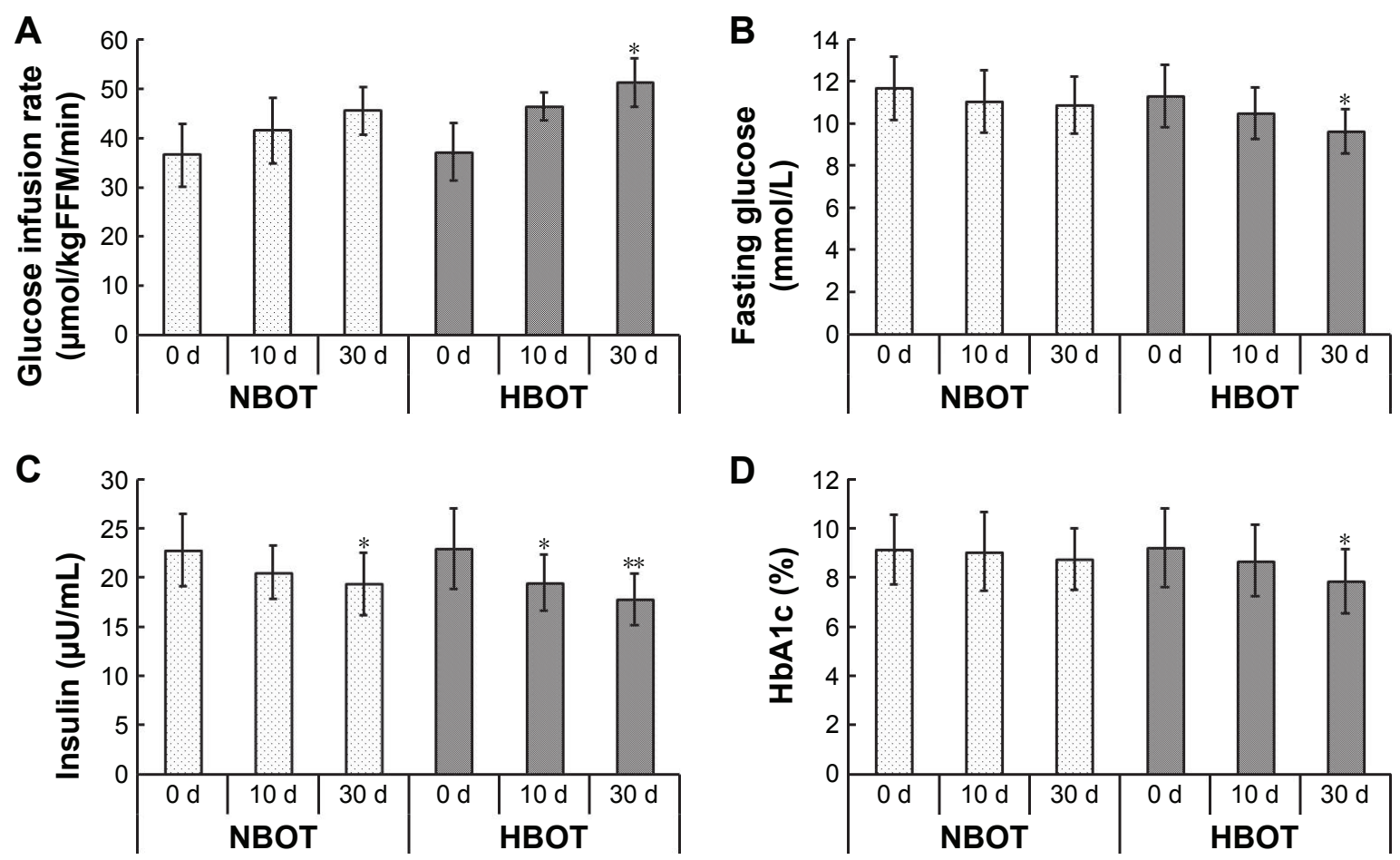

Figure 2 The influence of HBOT $(n=23)$ and NBOT $(n=29)$ on $(\mathbf{A})$ glucose infusion rate; (B) fasting glucose; (C) insulin; (D) hemoglobin $A I_{C}$ in type 2 diabetes patients suffering from intracerebral hemorrhage.

Notes: All data were presented as mean \pm standard deviation. Compared to the value before treatment, $* P<0.05$, $* * P<0.01$.

Abbreviations: NBOT, normobaric oxygen therapy; HBOT, hyperbaric oxygen therapy; d, days.
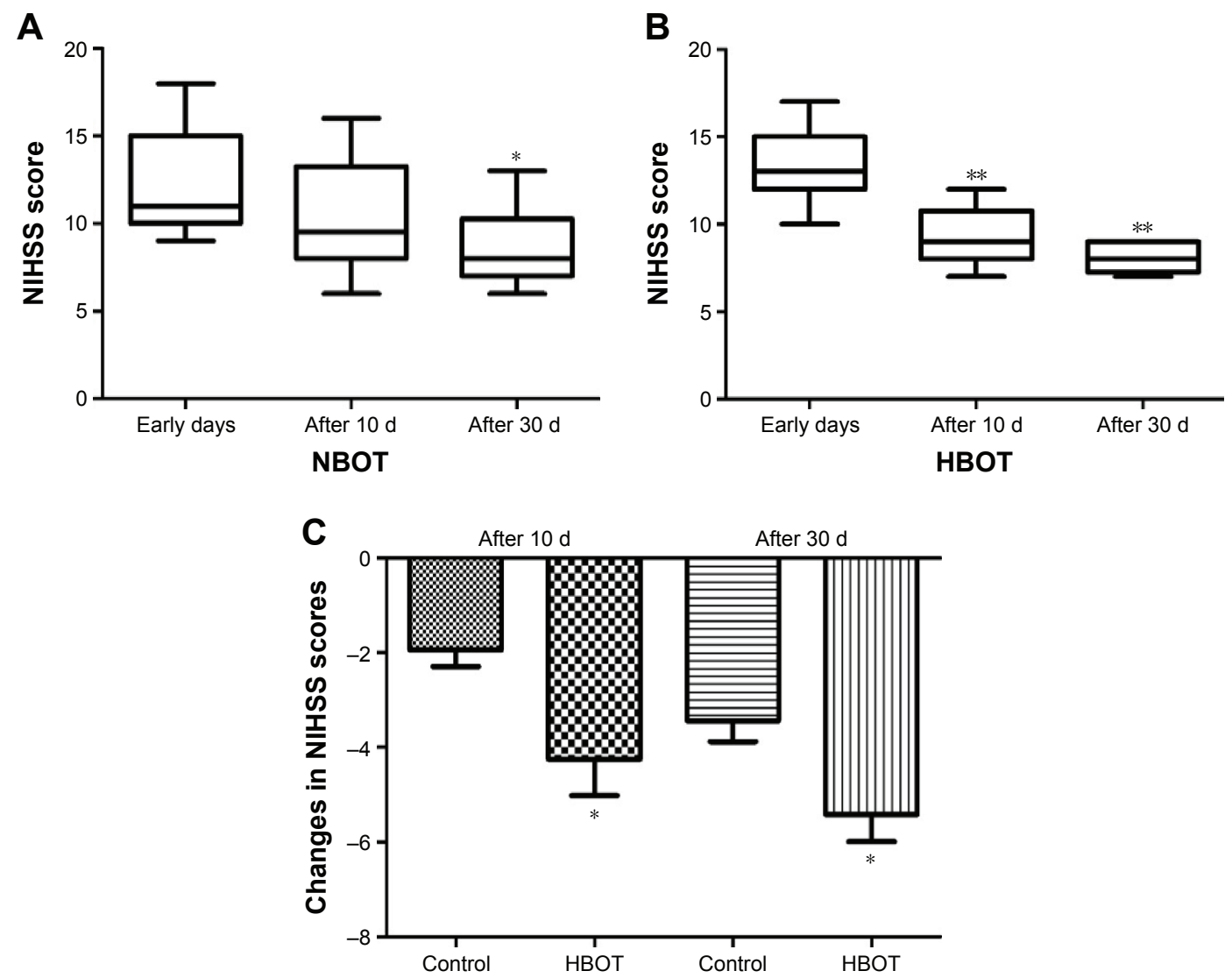

Figure 3 The clinical outcome (NIHSS score) in the NBOT control (A) and HBOT group (B); (C) the change in NIHSS scores in both groups. Note: $* P<0.05, * * P<0.01$.

Abbreviations: NIHSS, National Institutes of Health Stroke Scale; NBOT, normobaric oxygen therapy; HBOT, hyperbaric oxygen therapy; d, days. 
the HBOT group and the control group during the entire treatment period was not statistically significant $(P>0.05)$. However, the efficacy of HBOT in both early and late outcomes resulted in a statistically significant difference between the groups $(P<0.05$; Figure $3 \mathrm{C})$.

\section{Discussion}

The results of this study suggest that HBOT at 2.5 ATA for $1 \mathrm{~h}$ once a day in diabetes patients with intracerebral hemorrhage seems to be efficacious and beneficial in improving insulin sensitivity, and the efficacy of therapy is more obvious later in the disease course. A comparison of HBOT before and after treatment revealed that the HBOT group had better outcome in a long-term intervention. All patients in the HBOT group showed improvement, except for one patient who presented with worse outcome after 10 repetitions of HBOT (NIHSS score from 10 to 12), but no more disease progression after 1 month (NIHSS score from 12 to 12). Although the early efficacy of HBOT did not reach statistical significance, it still did reveal the tendency of better outcome compared to the control group. A few possible reasons could be explained as follows: 1) the mechanisms of HBOT are to (ie, protection of the blood-brain barrier) repair and generate new blood vessels for the injured part of brain and to improve metabolism after stroke. ${ }^{13}$ It is a long-time effect.

2) Cerebral edema occurs directly after stroke onset and peaks at $\sim 24-96 \mathrm{~h}$. It decreases blood flow and influences oxygen delivery. ${ }^{14} 3$ ) Since HBOT is an adjunctive treatment for stroke, patients during the acute stage commonly receive other treatments such as anti-inflammation therapy and/or antioxidants. Therefore, HBOT may not show its adjunctive effect significantly in mild stroke. 4) Finally, the patients included in this study are of mild severity. The degree of improvement of moderate-to-severe stroke may be more obvious than that of mild one.

As an interventional therapy, it is difficult to identify whether HBOT is more beneficial for patients with stroke only or for patients with both diabetes and stroke. It was observed that as a strong negative prognostic indicator in stroke, patients with admission hyperglycemia commonly showed poor clinical outcome with distinctly lower modified Rankin Scale scores and higher mortality compared to those without admission hyperglycemia. ${ }^{15}$ Another clinical trial reported that hemoglobin A1c level was relatively higher in acute ischemic stroke patients with diabetes and that the risk of mortality and poor outcome showed a tendency to increase with increasing HbA1c tercile. ${ }^{16}$ All we know currently is that a significant time effect was observed in the change in insulin sensitivity during the
HBOT sessions in both patients with or without diabetes, although significant reductions in fasting glucose during HBOT sessions were observed only in those with type 2 diabetes. $^{17}$

The major limitation was that this study was designed as a pilot study with a small number of patients, making its generalizability difficult. Although we originally proposed to enroll more patients in each group, the large workload and caseload limitation finally made us change the proposal.

There are several possible explanations for the results presented in this study. Diabetes patients with intracerebral hemorrhage may benefit from being treated within $24 \mathrm{~h}$ of symptom onset, considering the short therapeutic time window. Other explanations may be that the treating period applied in this study was relatively longer than that in other studies. ${ }^{7,11,12}$ A previous study showed that insulin sensitivity in overweight men with type 2 diabetes was improved following HBOT. ${ }^{13}$ Another study presumed that hyperbaric oxygen might decrease blood glucose levels in patients with diabetes mellitus, stroke, or traumatic brain injury during treatment. ${ }^{14}$ However, all recent studies were based on animal studies or were pilot trials with a small sample size. In animal model studies, HBOT with 2.5 ATA resulted in decreased lipid peroxidation, decreased leukocyte activation, ${ }^{2}$ and improved integrity of the blood-brain barrier. ${ }^{3}$

\section{Conclusion}

Our protocol of HBOT at 2.5 ATA was safe and feasible and appeared to be an efficacious treatment for acute stroke. Based on these results, a study on HBOT with a larger sample size is warranted.

\section{Acknowledgments}

This study was supported by a scientific project of Zhejiang province (no 2015KYA209). The authors acknowledge the HBOT nurses team for their personal time and commitment and the help from all colleagues in our hospital.

\section{Disclosure}

The authors report no conflicts of interest in this work.

\section{References}

1. Mink RB, Dutka AJ. Hyperbaric oxygen after global cerebral ischemia in rabbits does not promote brain lipid peroxidation. Crit Care Med. 1995;23(8):1398-1404.

2. Thom SR. Functional inhibition of leukocyte B2 integrins by hyperbaricoxygen in carbon monoxide-mediated brain injury in rats. Toxicol Appl Pharmacol. 1993;123(2):248-256.

3. Mink RB, Dutka AJ. Hyperbaric oxygen after global cerebral ischemia in rabbits reduces brain vascular permeability and blood flow. Stroke. 1995;26(12):2307-2312. 
4. Soejima Y, Hu Q, Krafft PR, Fujii M, Tang J, Zhang JH. Hyperbaric oxygen preconditioning attenuates hyperglycemia-enhanced hemorrhagic transformation by inhibiting matrix metalloproteinases in focal cerebral ischemia in rats. Exp Neurol. 2013;247:737-743.

5. Bian $\mathrm{H}, \mathrm{Hu} \mathrm{Q}$, Liang $\mathrm{X}$, et al. Hyperbaric oxygen preconditioning attenuates hemorrhagic transformation through increasing PPAR $\gamma$ in hyperglycemic MCAO rats. Exp Neurol. 2015;265:22-29.

6. Qin Z, Karabiyikoglu M, Hua Y, et al. Hyperbaric oxygen-induced attenuation of hemorrhagic transformation after experimental focal transient cerebral ischemia. Stroke. 2007;38(4):1362-1367.

7. Weinstein PR, Anderson GG, Telles DA. Results of hyperbaric oxygen therapy during temporary middle cerebral artery occlusion in unanesthetized cats. Neurosurgery. 1987;20(4):518-524.

8. Xue L, Yu Q, Zhang H, Liu Y, Wang C, Wang Y. Effect of large dose hyperbaric oxygenation therapy on prognosis and oxidative stress of acute permanent cerebral ischemic stroke in rats. Neurol Res. 2008; 30(4):389-393.

9. Chen SY, Huang E, Wang V, Fan YM, Ho CF, Yip PK. Improvement of clinical outcome and cerebral perfusion in a patient of atherosclerotic cerebral infarction after repetitive hyperbaric oxygen treatment - a case report and literature review. Undersea Hyperb Med. 2011;38(5):375-379.

10. Nighoghossian N, Trouillas P, Adeleine P, Salord F. Hyperbaric oxygen in the treatment of acute ischemic stroke: a double-blind pilot study. Stroke. 1995;26(8):1369-1372.
11. Taher A, Pilehvari Z, Poorolajal J, Aghajanloo M. Effects of normobaric hyperoxia in traumatic brain injury: a randomized controlled clinical trial. Trauma Mon. 2016;21(1):e26772.

12. Rockswold SB, Rockswold GL, Zaun DA, Liu J. A prospective, randomized phase II clinical trial to evaluate the effect of combined hyperbaric and normobaric hyperoxia on cerebral metabolism, intracranial pressure, oxygen toxicity, and clinical outcome in severe traumatic brain injury. J Neurosurg. 2013;118(6):1317-1328.

13. Zhang JH, Lo T, Mychaskiw G, Colohan A. Mechanisms of hyperbaric oxygen and neuroprotection in stroke. Pathophysiology. 2005; 12(1):63-77.

14. Katzman R, Clasen R, Klatzo I, Meyer JS, Pappius HM, Waltz AG. Report of Joint Committee for Stroke Resources. IV. Brain edema in stroke. Stroke. 1977;8(4):512-540.

15. Zuurbier SM, Hiltunen S, Tatlisumak T, et al. Admission hyperglycemia and clinical outcome in cerebral venous thrombosis. Stroke. 2016;47(2): 390-396.

16. Lei C, Wu B, Liu M, Chen Y. Association between hemoglobin $\mathrm{A}_{1} \mathrm{C}$ levels and clinical outcome in ischemic stroke patients with or without diabetes. J Clin Neurosci. 2015;22(3):498-503.

17. Wilkinson D, Nolting M, Mahadi MK, Chapman I, Heilbronn L. Hyperbaric oxygen therapy increases insulin sensitivity in overweight men with and without type 2 diabetes. Diving Hyperb Med. 2015;45(1): $30-36$.
Neuropsychiatric Disease and Treatment

\section{Publish your work in this journal}

Neuropsychiatric Disease and Treatment is an international, peerreviewed journal of clinical therapeutics and pharmacology focusing on concise rapid reporting of clinical or pre-clinical studies on a range of neuropsychiatric and neurological disorders. This journal is indexed on PubMed Central, the 'PsycINFO' database and CAS,

\section{Dovepress}

and is the official journal of The International Neuropsychiatric Association (INA). The manuscript management system is completely online and includes a very quick and fair peer-review system, which is all easy to use. Visit http://www.dovepress.com/testimonials.php to read real quotes from published authors. 